\title{
CARACTERIZAÇÃO DA CAPACIDADE FUNCIONAL, NIVEL COGNITIVO E FORÇA MUSCULAR RESPIRATÓRIA DE IDOSAS COM SINDROME PARKINSONIANA
}

\author{
Caren Schlottfeldt Fleck \\ Laís Rodrigues Gerzson² \\ Eduardo Matias dos Santos Steidl \\ Nathaly Marin Hernandez ${ }^{4}$ \\ resumo
}

Este estudo teve como objetivo caracterizar uma população idosa asilar com síndrome parkinsoniana quanto à força muscular respiratória, nível cognitivo e independência nas atividades de vida diária. $\bigcirc$ estudo caracterizou-se como uma série de casos prospectivos, na qual a amostra foi constituída por sete idosas diagnosticadas com síndrome parkinsoniana. $\bigcirc$ estado cognitivo das participantes foi avaliado por meio do Mini-Exame de Estado Mental, a capacidade

\footnotetext{
1 Fisioterapeuta (UNICRUZ), mestre em Ciências Médicas (UFRGS), professora do curso de Fisioterapia do Centro Universitário Franciscano (UNIFRA).E-mail: carenfleck@hotmail.com

2 Fisioterapeuta (UNIFRA). E-mail: gerzson.lais@yahoo.com.br

3 Fisioterapeuta (UNIFRA), mestrando em Distúrbios da Comunicação Humana (UFSM). E-mail: edumatias2005@gmail.com

4 Fisioterapeuta (UNIFRA), especializanda em Terapia Intensiva: Ênfase em Oncologia e Controle de Infecção Hospitalar (UNIFRA) e em Reabilitação Físico-Motora (UFSM). E-mail: nathalymh@hotmail.com
} 
funcional pelo Índice de Katz e a força muscular respiratória (inspiratória e expiratória) por manovacuometria. Os resultados deste estudo demonstraram que, em relação ao nível cognitivo, 71,44\% das idosas apresentaram perda cognitiva moderada; na capacidade funcional, a maioria apresentou independência parcial $(57,14 \%)$ e a força muscular respiratória se apresentou abaixo do esperado. Através dos resultados, foi possível concluir que, além do próprio envelhecimento, a SP pode ter influenciado de maneira negativa os aspectos cognitivos, a força muscular respiratória e a capacidade funcional das idosas avaliadas.

palavras - chave

Envelhecimento. Síndrome Parkinsoniana. Desempenho Funcional.

\section{Introdução}

Um dos fenômenos que tem chamado atenção na sociedade é o extraordinário crescimento da população acima dos 60 anos, tanto nos países em desenvolvimento quanto nos desenvolvidos (TRINDADE et al., 2011), fato este relacionado ao declínio nas taxas de fecundidade e de mortalidade (CARDOSO et al., 2012). Nas grandes cidades e nos países desenvolvidos, cerca de $20 \%$ da população estaria enfrentando modificações que ocorrem na terceira idade, como a fragilidade inerente ao envelhecimento, a qual traz um aumento da demanda de cuidados intensivos e contínuos (MENEZES; VICENTE, 2007).

A Organização Mundial da Saúde (OMS) define idoso como aquele que tem 60 anos ou mais; fisiologicamente, contudo, não há um marco para o início da senescência. O envelhecimento surge associado com uma série de modificações orgânicas, com declínio das capacidades funcionais e alterações no funcionamento fisiológico de órgãos e sistemas. Importante salientar que, apesar da predisposição a desenvolver doenças, o envelhecimento não pode ser considerado sinônimo de adoecimento, especialmente quando o idoso desenvolve hábitos de vida saudáveis (STELLA et al., 2002; TRINDADE et al., 2011).

Os declínios das funções sistêmicas do organismo durante o envelhecimento estão relacionados a aspectos funcionais e psíquicos; no primeiro caso estão envolvidos inputs sensoriais e de reações (automáticas, reflexas e 
voluntárias) motoras, e no segundo, alterações cognitivas, que repercutem sobre atividades básicas e instrumentais de vida diária do idoso (MEIRELES et al., 2010).

Neste contexto, o parkinsonismo, ou síndrome parkinsoniana (SP) ${ }^{5}$, é um complexo de sinais e sintomas cardinais, os quais acometem o idoso, caracterizado pela presença de tremor de repouso, rigidez muscular, bradicinesia, instabilidade postural e, frequentemente, comprometimento cognitivo (FERRAZ; MOURÃO, 2003), decorrentes de alterações na via dopaminérgica nigro-estriatal (COSTA et al., 2003; SOUZA et al., 2011). Conforme apontado, a palavra parkinsonismo é usada para descrever um conjunto de sinais e sintomas relacionados a desordens do movimento (SANT et al., 2008).

Em relação à etiologia, a SP pode ser dividida em três categorias: parkinsonismo primário ou Doença de Parkinson (DP), secundário (de causa reconhecida, como o uso de drogas, intoxicações e acometimentos do sistema nervoso central) e parkinsonismo-plus (grupo de doenças neurodegenerativas que apresentam manifestações neurológicas habitualmente não encontradas na DP) (COSTA et al., 2003; STEIDL et al., 2007).

Em virtude das diferentes formas de apresentação do parkinsonismo, há uma dificuldade em predizer a prevalência e a incidência da síndrome. Porém, estudos têm demonstrado que a DP, por ser a manifestação clínica mais comum da SP, tem se tornado um problema para a sociedade, sendo capaz de provocar impactos nas estruturas econômicas, sociais e de saúde (STEIDL et al., 2007). Acredita-se que a prevalência de idosos com DP no Brasil, com idades entre 60 e 69 anos, atinja 700/100.000, e entre 70 e 79 anos, 1.500/100.000, surgindo a cada ano 36 mil novos casos (IKE et al., 2008; SOUZA et al., 2011). Ainda, estima-se que em 2020, aproximadamente 40 milhões de pessoas no mundo terão desordens motoras secundárias à DP (LANA et al., 2007).

Em decorrência da cronicidade e das diversas manifestações clínicas e funcionais presentes nos portadores de parkinsonismo, avaliações que visem conhecer e diagnosticar alterações presentes nesses indivíduos, tais como das atividades de vida diária, nível cognitivo e função muscular respiratória, tornam-se de extrema importância a fim de estabelecer estratégias terapêuticas e amenizar possíveis comprometimentos, melhorando a qualidade de vida.

Baseando-se nessas premissas, o presente estudo propôs caracterizar uma população idosa asilar, com SP, quanto à força muscular respiratória, nível cognitivo e independência nas atividades de vida diária.

5 A SP é caracterizada pelos sinais de tremor de repouso, rigidez muscular, bradicinesia e instabilidade postural, em decorrência de uma disfunção da via dopaminérgica nigro-estriatal. 
Este estudo caracterizou-se como uma série de casos prospectivos, realizado na Instituição de Longa Permanência para Idosos (ILPI) Associação Amparo Providência Lar das Vovozinhas, na cidade de Santa Maria - RS. A pesquisa foi aprovada pelo Comitê de Ética em Pesquisa (CEP) do Centro Universitário Franciscano (UNIFRA) sob parecer no 294.2010.2, e ocorreu somente após a assinatura do Termo de Consentimento Livre e Esclarecido (TCLE) pelas participantes.

A amostra foi por conveniência, onde foram estudadas idosas com diagnóstico neurológico de SP que concordaram participar do estudo e assinaram espontaneamente o TCLE. Foram excluídas idosas com incapacidade de compreensão, tabagistas e com doenças pulmonares prévias, além disso, os dados foram coletados uma hora após administração da medicação utilizada (Levodopa), a fim de evitar tremores de mandíbula e, consequentemente, escape aéreo na avaliação da força muscular respiratória. Após a aplicação dos critérios de exclusão, sete idosas fizeram parte da amostra.

O estado cognitivo das participantes foi verificado por meio da aplicação do Mini-Exame do Estado Mental (MEEM), sendo este um breve questionário de 30 pontos que avalia perda cognitiva. A pontuação igual ou superior a 25 representa normalidade; de 21 a 24 pontos, perda leve; de 10 a 20 pontos, moderada; e abaixo de 9, perda cognitiva grave (FOLSTEIN et al., 1975). Após, foi aplicada uma ficha de avaliação, através da qual foram coletadas informações referentes aos dados pessoais e clínicos das participantes.

A capacidade funcional das idosas foi verificada pelo Índice de Katz (KATZ et al., 1963). Esse instrumento avalia a independência no desempenho de seis funções (banho, vestir-se, ir ao banheiro, transferência ${ }^{6}$, continência e alimentação), classificando as pessoas idosas como independentes ou dependentes. O Índice de Katz, devido à praticidade de sua aplicação e sua confiabilidade, demonstradas em estudos semelhantes, mostra-se um dos instrumentos mais adequados para a avaliação da capacidade funcional, pois é validado para a população brasileira e beneficia a avaliação tanto de indivíduos idosos quanto de doentes crônicos e daqueles em longos períodos de recuperação hospitalar. Pode ser utilizado, ainda, para avaliar a capacidade funcional do indivíduo na comunidade.

6 Capacidade do idoso em transferir-se da cama para a posição ortostática, com auxílio ou não, para a realização das demais atividades de vida diária. 
Outra avaliação realizada foi da força muscular respiratória por meio da medida da pressão inspiratória máxima (PImáx) e da pressão expiratória máxima (PEmáx), cujas mensurações foram feitas por meio de um manovacuômetro analógico marca Microhard MVD 300, com intervalo operacional de $-150 \mathrm{a}+150 \mathrm{cmH}_{2} \mathrm{O}$, com o paciente sentado. Foi adaptado ao paciente um clipe nasal para evitar escape aéreo.

Para mensurar a PImáx, foi solicitado à idosa que iniciasse uma inspiração a partir do volume residual (VR), isto é, após uma expiração máxima. A inspiração deveria durar pelo menos três segundos com o máximo de esforço e tempo possíveis (CADER et al., 2007). Para a mensuração da PEmáx, a idosa foi orientada a expirar a partir da capacidade pulmonar total (CPT), ou seja, após uma inspiração profunda, devendo durar no mínimo três segundos com o máximo de força e tempo possíveis. Cada mensuração foi repetida de três a cinco manobras máximas, aceitáveis e reprodutíveis (diferença de 10\% ou menos entre os esforços), com intervalo de descanso entre os esforços de aproximadamente um minuto, sendo registrado o valor mais alto. As participantes foram orientadas a evitar o colapso das bochechas durante a mensuração e, desta forma, a elevação da pressão da cavidade oral, gerada exclusivamente por contração da musculatura facial com fechamento da glote. As equações propostas por Neder et al. (1999), nas quais a idade é levada em consideração, foram utilizadas para o cálculo dos valores previstos da PImáx e PEmáx.

Os dados coletados foram processados na planilha eletrônica do programa Microsoft Excel 2007, e os resultados apresentados na forma de frequência, porcentagem, média e desvio padrão.

\section{Resultados}

O grupo estudado foi composto por sete idosas com valores de idade que variaram de 64 a 91 anos, uma média de 73ะ8,62 anos. Na avaliação da capacidade funcional, os resultados encontrados demonstraram que 57,14\% $(n=4)$ das idosas foram classificadas como parcialmente independentes, ou seja, recebiam algum tipo de auxílio principalmente no banho e vestuário e apresentavam perdas urinárias e/ou fecais acidentais, 28,58\% ( $\mathrm{n}=2)$ como independentes e $14,28 \%(\mathrm{n}=1)$ como totalmente dependentes.

O nível cognitivo das idosas demonstrou que $14,28 \%(n=1)$ apresentou intelecto preservado, $14,28 \%(n=1)$ possuía perda leve e $71,44 \%(n=5)$, perda cognitiva moderada. As que possuíam perda leve apresentaram pior 
desempenho no item "Atenção ao cálculo" e as moderadas nos itens "Registro", "Atenção ao cálculo" e "Memória".

Em relação à força muscular respiratória, as idosas apresentaram média de PImáx e PEmáx de 37,85 $\pm 14,09 \mathrm{mmHg}$ e 51,42 $\pm 25,44 \mathrm{mmHg}$, respectiva-

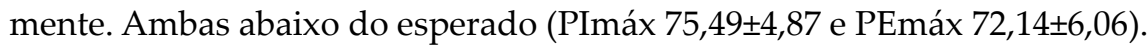

Os dados podem ser visualizados na tabela abaixo (Tabela 1).

Tabela 1 - Características das idosas estudadas.

\begin{tabular}{c|c|c|c|c|c}
\hline Idosa & Idade & $\begin{array}{c}\text { Índice de } \\
\text { Katz }\end{array}$ & MEEM & $\begin{array}{c}\text { PImáx } \\
\mathbf{( c m H 2 O})\end{array}$ & $\begin{array}{c}\text { PEmáx } \\
\mathbf{( c m H 2 O})\end{array}$ \\
\hline 1 & 69 & I & 20 & 60 & 30 \\
\hline 2 & 68 & Pl & 23 & 40 & 60 \\
\hline 3 & 69 & Pl & 15 & 45 & 90 \\
\hline 4 & 70 & I & 16 & 40 & 60 \\
\hline 5 & 80 & PI & 11 & 20 & 30 \\
\hline 6 & 91 & TD & 25 & 40 & 70 \\
\hline 7 & 64 & PI & 13 & 20 & 20 \\
\hline
\end{tabular}

I - independente; PI - parcialmente independente; TD - totalmente dependente; $\mathrm{CmH}_{2} \mathrm{O}$ - centímetros de água; MEEM - Mini-Exame do Estado Mental.

\section{Discussão}

Através dos resultados encontrados observa-se que $57,14 \%(\mathrm{n}=4)$ das idosas foram classificadas como parcialmente independentes, $28,58 \%(\mathrm{n}=2)$ como independentes e $14,28 \%(n=1)$ como totalmente dependentes. Através disso é possível verificar que, as idosas avaliadas apresentaram redução da capacidade funcional em suas atividades de vida diária (AVD's). O processo fisiológico do envelhecimento favorece limitações nestas atividades, em decorrência da fragilidade encontrada nesta população que em indivíduos com SP pode estar mais acentuada pelo comprometimento motor.

A fragilidade é definida pela Sociedade Americana de Geriatria como o declínio de reservas e resistência aos agentes estressores, os quais agem na homeostase do organismo, resultando no declínio de múltiplos sistemas fisiológicos, o qual repercute sobre a capacidade funcional, principalmente 
de idosos, afetando suas AVD's. Com a idade avançada, pode-se observar um declínio na capacidade funcional do indivíduo, os idosos, especificamente, podem tornar-se portadores de fragilidades que influenciam na sua funcionalidade e, consequentemente, afetam o desempenho em suas AVD's. No que se refere à realização das mesmas, alguns estudos já evidenciaram que quanto maior a idade, maior pode ser o nível de dependência do idoso na execução dessas atividades (BONARDI et al., 2007).

Como exemplo, tem-se o estudo de Alves et al. (2008) realizado no município de Juiz de Fora (MG), no qual foram entrevistados 655 idosos, sorteados aleatoriamente na base do SIAB (Sistema de Informação da Atenção Básica), com o objetivo de conhecer suas características e avaliar sua capacidade funcional. Os resultados deste apontaram que 58,2\% dos idosos apresentaram grau de dependência e este percentual se elevou com o avanço da idade. Os achados permitiram aos autores observar que as atividades contidas nas AVD's tornam-se muito importantes na manutenção da capacidade funcional.

Em relação ao impacto da SP na capacidade funcional de idosos, Goulart et al. (2004) verificaram que nos indivíduos com DP o desempenho funcional sofre impacto desde fases iniciais da doença e não somente na avançada.

Durante o envelhecimento, várias doenças podem acometer o idoso, de forma a interferir na sua saúde e capacidade funcional. Tais doenças, crônicas ou degenerativas, exigem uma ação multiprofissional em conjunto, que vise às necessidades de cada idoso, de forma a propiciar a manutenção e o aperfeiçoamento de sua funcionalidade (LEVY, 2001; REICHEL; GALLO, 2001).

A partir de uma abordagem diagnóstica e terapêutica multidimensionais, ou seja, dispensando uma atenção abrangente à saúde do idoso de forma interdisciplinar e planejada, as doenças crônicas são controladas e as agudas, tratáveis, preservando-se, assim, a autonomia e a independência e promovendo uma melhor qualidade de vida ao idoso (PAPALÉO NETTO; KLEIN; BRITO, 2005).

A avaliação da capacidade funcional dos idosos tem se tornado cada vez mais importante, tanto no aspecto preventivo, quanto na assistência em níveis secundários, nos quais já se percebe a necessidade de tratamento do indivíduo. A capacidade de manter as habilidades físicas e mentais necessárias para uma vida independente e autônoma é um dos conceitos dados à capacidade funcional. Do ponto de vista da Saúde Pública define-se a capacidade funcional como um novo conceito de saúde, mais adequado para instrumentalizar e operacionalizar a atenção à saúde do idoso (GORDILHO et al., 2000). 
O comprometimento cognitivo é comum na população idosa em decorrência do processo de envelhecimento. Estudos demonstram associação do déficit cognitivo com graus variados de dependência funcional (CASTRO; GUERRA, 2008; LAKS et al., 2005).

Neste estudo, em relação aos resultados para o MEEM nas idosas avaliadas, $14,28 \%(n=1)$ apresentou intelecto preservado, $14,28 \%(n=1)$ possuía perda leve e $71,44 \%(n=5)$ perda cognitiva moderada. Este resultado nos mostra que a maior parte das idosas apresenta uma perda cognitiva moderada, quando nos resultados da capacidade funcional temos a maior parte das idosas apresentando uma independência parcial.

Com relação à função cognitiva, segundo Galhardo et al. (2009), em um levantamento bibliográfico, os estudos que relacionam as funções cognitivas e a DP demonstram alterações na memória, linguagem, capacidade visuoespacial e funções executivas, caracterizando a DP como uma demência, que muitas vezes irá manifestar os seus sintomas no decorrer de alguns anos após o paciente ser diagnosticado.

A institucionalização, por si só, representa um fator de risco, já que os idosos institucionalizados necessitam de atenção, suporte social e serviços especializados, pois a grande maioria é fragilizada e apresenta morbidades físicas e/ou mentais (PAULO et al., 2012).

Devido ao seu isolamento social, pela inatividade física e processos psicológicos, subentende-se que quanto maior o tempo de institucionalização, maior o comprometimento da saúde e vida do idoso, estas entre as perdas mais significativas (SANTOS; ANDRADE, 2005).

Dentre os vários comprometimentos acometidos a esses idosos institucionalizados, a capacidade funcional, especialmente a dimensão motora, é um dos importantes marcadores de um envelhecimento bem sucedido e de qualidade de vida. A perda dessa capacidade está associada à predição de fragilidade e dependência, com riscos de quedas e problemas de mobilidade, complicações ao longo do tempo, necessidades de cuidados especiais de alto custo a longo prazo e até mesmo à morte (GIACOMIN et al., 2008).

Para a Organização Mundial da Saúde (OMS) (1998), a participação em atividades físicas leves e moderadas pode retardar os declínios funcionais, assim, uma vida ativa melhora a saúde mental e contribui na gerência de desordens como a depressão e a demência. Existem evidências que idosos fisicamente ativos apresentam menor prevalência de doenças mentais do que os não-ativos.

Diversos estudos referem-se aos benefícios da atividade física para todas as idades, principalmente durante o processo de envelhecimento, não 
somente em relação ao bem-estar, mas, também, à melhora da capacidade física, tanto na prevenção e minimização dos efeitos deletérios do envelhecimento, quanto nos sintomas psiquiátricos dos mesmos (MAZO et al., 2006).

A atividade física possibilita uma dinâmica corporal mais ativa a fim de evitar as perdas do condicionamento físico e o sedentarismo e dar um sentido para os momentos de convivência nesses lares. Ademais, com a prática regular do exercício físico funcional, poder-se minimizar o uso de medicamentos, possibilitar ao idoso uma movimentação mais ampla no ambiente de acolhimento e, ainda, ampliar o seu convívio com outras pessoas fora da instituição acolhedora (PAULO et al., 2012).

O movimento orientado e trabalhado sistematicamente com os idosos é essencial para garantir uma qualidade de vida mais apropriada para idosos institucionalizados, pois melhora as suas capacidades físicas funcionais e os integra novamente às suas tarefas cotidianas, o que possibilita sua reintegração social (PAULO et al., 2012).

Outro ponto importante encontrado no estudo foi o declínio da força muscular respiratória, tanto da PImáx, quanto da PEmáx nas idosas avaliadas. Os valores encontrados no estudo foram, em média, PImáx e PEmáx de $37,85 \pm 14,09 \mathrm{mmHg}$ e $51,42 \pm 25,44 \mathrm{mmHg}$, respectivamente. Ambas abaixo do

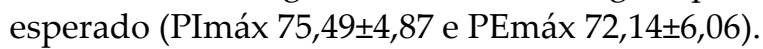

$\mathrm{O}$ declínio da força muscular respiratória possui relações diretas com o processo do envelhecimento, do qual o enrijecimento dos arcos costais, déficit na força muscular, diminuição da densidade óssea, menor fluxo sanguíneo cerebral, atrofia muscular, menor excursão diafragmática e redução dos volumes e capacidades pulmonares interferem diretamente na força da musculatura respiratória e na restrição da ventilação; como consequência, há o comprometimento direto das atividades de vida diária (FANÒ et al., 2001; FERREIRA et al., 2009).

Além disto, ocorrem reduções da mobilidade da caixa torácica, da elasticidade pulmonar e dos valores da pressão inspiratória máxima, o que, consequentemente, levará a uma redução da eficiência de tosse, bem como a diminuição da mobilidade dos cílios do epitélio respiratório (FRANCISCO et al., 2006).

A postura corporal, em especial do portador de SP é caracterizada como postura em flexão (hiperlordose cervical), devido às desordens nos sistemas vestibulares, visuais e proprioceptivos, onde tendem a se deslocar para frente. Pode, ainda, comprometer a prosódia (FERREIRA et al., 2007) e as vias respiratórias devido à perda de flexibilidade da musculatura respiratória. 
Devido ao vetor de gravidade para frente, é provocada a chamada marcha festinada, ou marcha rápida (PARREIRA et al., 2003), onde os músculos adutores e abdutores tornam-se mais contraídos, tanto nos membros superiores como nos inferiores. Esta postura pode não ser notada no início da doença, mas com seu progresso torna-se perceptível, acarretando desordens na caixa torácica e, por conseqüência, na musculatura respiratória (MATA; BARROS; LIMA, 2008).

\section{Considerações finais}

Através deste estudo, foi possível concluir que além do próprio envelhecimento, a SP pode ter influenciado ainda mais os aspectos cognitivos, respiratórios e a capacidade funcional das idosas institucionalizadas. Sendo assim, sugere-se que se façam mais estudos com estes parâmetros, a fim de verificar uma possível relação entre nível cognitivo, força muscular respiratória e capacidade funcional em idosas com SP.

Além disso, uma abordagem multiprofissional nas Instituições de Longa Permanência para Idosos, através de atividades físicas e de recreação que favoreçam a preservação do intelecto, das AVD's e, consequentemente, da força muscular respiratória, tendendo a minimizar os efeitos do próprio envelhecimento e da SP.

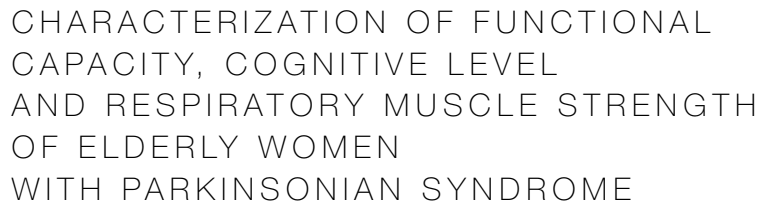

This study aim was to characterize an asylum elderly population with Parkinsonian Syndrome regarding their respiratory muscle strength, cognitive level and independence in activities of daily living. The study was characterized as a series of prospective cases, in which the sample was composed of seven elderly women diagnosed with Parkinsonian Syndrome. The cognitive status of the participants was assessed through the Mini-Mental State Examination, the functional capacity by the Katz Index and the respiratory muscle strength 
(inspiratory and expiratory) by manovacuometry. The results of this study showed that in relation to the cognitive level, $71.44 \%$ of the elderly had mild cognitive impairment; in functional capacity, the majority showed partial independence $(57.14 \%)$ and the respiratory muscle strength performed below expectations. Through the results, one might conclude that, in addition to aging itself, the PS may have influenced negatively the cognitive aspects, the respiratory muscle strength and the functional capacity of the assessed elderly.

keywords

Aging. Parkinsonian Syndrome. Functional Performance.

referências

ALVES, Márcio José Martins et al. Perfil da Capacidade Funcional do Idoso. In: ENCONTRO NACIONAL DE ESTUDOS POPULACIONAIS, 16., 2008, Caxambu. Anais... Caxambu: ABEP, 2008. p 1-18.

BONARDI, Gislaine et al. Incapacidade funcional e idosos: um desafio para os profissionais de saúde. Scientia Medica, Porto Alegre, v. 17, n. 3, p. 138-144, jul./set. 2007.

CADER, Samária et al. Efeito do treino dos músculos inspiratórios sobre a pressão inspiratória e a autonomia funcional de idosos asilados. Motricidade, Vila Real, v. 3, n. 1, p. 279-288, jan. 2007.

CARDOSO, Maria Cristina et al. Análise da capacidade funcional dos idosos de Porto Alegre e sua associação com autopercepção de saúde. Estudos Interdisciplinares sobre o Envelhecimento, Porto Alegre, v. 17, n. 1, p. 111-124, jun. 2012.

CASTRO, Keithlen Cruz Moreira de; GUERRA, Ricardo Oliveira. Impacto do desempenho cognitivo na capacidade funcional em uma amostra populacional de idosos do nordeste brasileiro. Arquivos de Neuro-Psiquiatria, São Paulo, v. 66, n. 4, p. 809-813, dez. 2008.

COSTA, Maria do Desterro Leiros da et al. Alterações de neuroimagem no parkinsonismo: estudo de cinco casos. Arquivos de Neuro-Psiquiatria, São Paulo, v. 61, n. 2-B, p. 381-386, jun. 2003.

FANO, Giorgio et al. Age and sex influence on oxidative damage and functional status in human skeletal muscle. Journal of Muscle Research and Cell Motility, London, v. 22, n. 4, p. 345-351, May 2001.

FERRAZ, Henrique Ballalai; MOURÃO, Lucia Figueiredo. Doença de Parkinson. In: CHIAPPEITA, Ana Lúcia de Magalhães Leal (Org.). Conhecimentos essenciais para atender bem o paciente com doenças neuromusculares, Parkinson e Alzheimer. São José dos Campos: Pulso, 2003. p. 45-54.

FERREIRA, Fernanda Vargas et al. Doença de Parkinson - Força muscular respiratória e atividades de vida diária: relato de caso. Revista Congrega Urcamp, Bagé, n. 3, p. $21-30,2009$.

A relação da postura corporal com a prosódia na Doença de Parkinson: estudo de caso. Revista CEFAC, São Paulo, v. 9, n. 3, p. 319-329, jul./set. 2007. 
FOLSTEIN, Marshal F. et al. Mini-Mental State: a practical method for grading the cognitive state of patients for the clinician. Journal of Psychiatric Research, Stanford, v. 12, n. 3, p. 189-198, nov. 1975

FRANCISCO, Priscila Maria Stolses Bergamo et al. Fatores associados à doença pulmonar em idosos. Revista de Saúde Pública, São Paulo, v. 40, n. 3, p. 428-435, jun. 2006

GALHARDO, Mônica Maria de Azevedo Mello Carvalho et al. Caracterização dos distúrbios cognitivos na doença de Parkinson. Revista CEFAC, São Paulo, v. 11, supl. 2, p. 251-257, 2009

GIACOMIN, Karla Cristina et al. Estudo de base populacional dos fatores associados à incapacidade funcional entre idosos na Região Metropolitana de Belo Horizonte, Minas Gerais, Brasil. Cadernos de Saúde Pública, Rio de Janeiro, v. 24, n. 6, p. 1260-1270, jun. 2008

GORDILHO, Adriano et al. Desafios a serem enfrentados no terceiro milênio pelo setor saúde na atenção integral ao idoso. Rio de Janeiro: Universidade Aberta da Terceira Idade - UnATI, 2000

GOULART, Fátima et al. Análise do desempenho funcional em pacientes portadores de doença de Parkinson. Acta Fisiátrica, São Paulo, v. 11, n. 1, p. 12-16, abr. 2004.

IKE, Daniela et al. Análise da incidência de quedas e a influência da fisioterapia no equilíbrio e na estabilidade postural de pacientes com doença de Parkinson. Revista Fisioterapia Brasil, Rio de Janeiro, v. 9, n. 1, p. 4-8, jan./fev. 2008.

KATZ, Sidney et al. Studies of illness in the aged. The index of ADL: a standardized measure of biological and psychosocial function. JAMA: The Journal of The American Medical Association, Chicago, v. 185, n. 12, p. 914-919, Sept. 1963.

LAKS, Jerson et al. Prevalence of cognitive and functional impairment in community-dwelling elderly: importance of evaluating activities of daily living. Arquivos de Neuro-Psiquiatria, São Paulo, v. 63, n. 2a, p. 207-212, jun. 2005

LANA, R. C. et al. Percepção da Qualidade de Vida de indivíduos com doença de Parkinson através do PDQ-39. Revista Brasileira de Fisioterapia, São Carlos, v. 11, n. 5 , p. 397-402, set./out. 2007

LEV, Susan M. Avaliação multidimensional do paciente idoso. In: GALLO, Joseph J. (Ed.) et al. Reichel - assistência ao idoso: aspectos clínicos do envelhecimento. Rio de Janeiro: Editora Guanabara Koogan, 2001. p. 15-29.

MATA, Fabiana Araújo Figueiredo da; BARROS, Alcidezio Luiz Sales; LIMA, Cláudia Fonseca. Avaliação do risco de quedas em pacientes com Doença de Parkinson. Revista Neurociências, São Paulo, v. 16, n. 1, p. 20-24, 2008

MAZO, Giovana Zarpellon et al. Programa de hidroginástica para idosos: motivação, auto-estima e auto-imagem. Revista Brasileira de Cineantropometria \& Desempenho Humano, Florianópolis, v. 8, n. 2, p. 67-72, jun. 2006

MEIRELES, Aline Estrela et al. Alterações neurológicas fisiológicas ao envelhecimento afetam o sistema mantenedor do equilibrio. Revista Neurociências, São Paulo, v. 18, n. 1. p. 103-108, 2010.

MENEZES, Letícia Neiva; VICENTE, Laélia Cristina Caseiro. Envelhecimento vocal em idosos institucionalizados. Revista CEFAC, São Paulo, v. 9, n. 1, p. 90-98, jan./mar. 2007.

NEDER, José Alberto et al. Reference values for lung function tests. II. Maximal respiratory pressures and voluntary ventilation. Brazilian Journal of Medical and Biological Research, Ribeirão Preto, v. 32, n. 6, p. 719-727, jun. 1999. 
OMS - Organização Mundial da Saúde. Population aging: a public health challenge. Geneva: WHO Press Office, 1998.

PAPALÉO NETTO, Matheus; KLEIN, Eva Lucila; BRITO, Francisco Carlos de. Avaliação geriátrica multidimensional. In: CARVALHO FILHO, Eurico Thomaz de; PAPALÉO NEITO, Matheus. Geriatria: fundamentos, clínica e terapêutica. São Paulo: Atheneu, 2005. p. 73-85.

PARREIRA, Verônica Franco et al. Padrão respiratório em pacientes portadores da doença de Parkinson e em idosos assintomáticos. Acta Fisiátrica, São Paulo, v. 10, n. 2, p. 61-66, ago. 2003.

PAULO, Thaís Reis Silva de et al. O exercício físico funcional para idosos institucionalizados: um novo olhar para as atividades da vida diária. Estudos Interdisciplinares sobre o Envelhecimento, Porto Alegre, v. 17, n. 2, p. 413-427, dez. 2012.

REICHEL, William; GALLO, Joseph J. Princípios fundamentais da assistência ao idoso. In: GALLO, Joseph J. (Ed.) et al. Reichel - assistência ao idoso: aspectos clínicos do envelhecimento. Rio de Janeiro: Guanabara Koogan, 2001. p. 3-14.

SANT, Cíntia Ribeiro de et al. Abordagem fisioterapêutica na Doença de Parkinson. Revista Brasileira de Ciências do Envelhecimento Humano, Passo Fundo, v. 5, n. 1, p. 80-89, jan./jun. 2008.

SANTOS, Milena Lisboa Couto dos; ANDRADE, Marinúbia Chaves de. Incidência de quedas relacionada aos fatores de riscos em idosos institucionalizados. Revista Baiana de Saúde Pública, Salvador, v. 29, n. 1, p. 57-68, jan./jun. 2005.

SOUZA, Cheylla Fabricia de Medeiros et al. A doença de Parkinson e o processo de envelhecimento motor: uma revisão de literatura. Revista de Neurociências, São Paulo, v. 19, n. 4, p. $718-723,2011$

STEIDL, Eduardo Matias dos Santos et al. Doença de Parkinson: revisão bibliográfica. Disciplinarum Scientia, Santa Maria, v. 8, n. 1, p. 115-129, 2007.

STELLA, Florindo et al. Depressão no idoso: diagnóstico, tratamento e benefícios da atividade física. Motriz, Rio Claro, v. 8, n. 3, p. 91-98, ago./dez. 2002.

TRINDADE, Thaís Marinho et al. Capacidade pulmonar de idosos praticantes de hidroginástica. Estudos Interdisciplinares sobre o Envelhecimento, Porto Alegre, v. 16, n. 1 , p. 79-96, jun. 2011. 